UCRHEP-T444

January 2008

\title{
Supersymmetric U(1) Gauge Realization of the Dark Scalar Doublet Model of Radiative Neutrino Mass
}

\author{
Ernest Ma \\ Department of Physics and Astronomy, University of California, \\ Riverside, California 92521, USA
}

\begin{abstract}
Adding a second scalar doublet $\left(\eta^{+}, \eta^{0}\right)$ and three neutral singlet fermions $N_{1,2,3}$ to the Standard Model of particle interactions with a new $Z_{2}$ symmetry, it has been shown that $\eta_{R}^{0}$ or $\eta_{I}^{0}$ is a good dark-matter candidate and seesaw neutrino masses are generated radiatively. A supersymmetric U(1) gauge extension of this new idea is proposed, which enforces the usual $R$ parity of the Minimal Supersymmetric Standard Model, and allows this new $Z_{2}$ symmetry to emerge as a discrete remnant.
\end{abstract}


A new idea has recently been proposed [1, 2, 3] that without dark matter, neutrinos would be massless. This is minimally implemented in the Standard Model (SM) of particle interactions by the addition of a second scalar doublet $\left(\eta^{+}, \eta^{0}\right)$ and three neutral singlet Majorana fermions $N_{1,2,3}$, together with a new $Z_{2}$ discrete symmetry [4], under which $\left(\eta^{+}, \eta^{0}\right)$ and $N_{1,2,3}$ are odd, and all SM particles are even. Using the allowed term $\left(\lambda_{5} / 2\right)\left(\Phi^{\dagger} \eta\right)^{2}+H . c$. , where $\Phi=\left(\phi^{+}, \phi^{0}\right)$ is the SM Higgs doublet, seesaw neutrino masses are generated in one loop, as shown in Fig. 1.

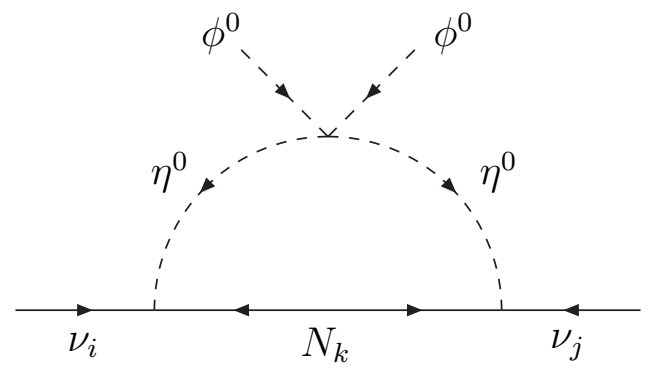

Figure 1: One-loop generation of neutrino mass.

At the same time, $\eta_{R}^{0}$ and $\eta_{I}^{0}$ are split in mass, and whichever is lighter has been shown to be a good dark-matter candidate [5, 6, 7] with mass between 45 and $75 \mathrm{GeV}$, with reasonable prognosis [5, 8] for detection at the Large Hadron Collider (LHC). Variants of this basic idea have also been discussed [9, 10, 11, 12, 13, 14, 15, 16, 17.

Consider now its supersymmetric generalization. The simplest version [3, 18] is to retain the proposed $Z_{2}$ symmetry in addition to the usual $R$ parity of the Minimal Supersymmetric Standard Model (MSSM) which forbids proton decay. Another is to use the string-inspired $E_{6} / U(1)_{N}$ model [19] but it is still necessary to impose the two $Z_{2}$ symmetries by hand. In either case, there are two kinds of dark matter as recently discussed [20, 21].

In this paper, instead of imposing the two $Z_{2}$ symmetries by hand, a new $\mathrm{U}(1)$ gauge symmetry is proposed, from which $R$ parity is automatically derived. Further, as this $\mathrm{U}(1)$ 
gauge symmetry is spontaneously broken, its residual $Z_{2}$ discrete symmetry [12] is exactly what is needed for radiative neutrino mass and the second type of dark matter.

Consider the following U(1) gauge extension of the MSSM with particle content as shown in Table 1.

Table 1: MSSM particle content of proposed model.

\begin{tabular}{|c|c|c|}
\hline Superfield & $S U(3)_{C} \times S U(2)_{L} \times U(1)_{Y}$ & $U(1)_{X}$ \\
\hline$Q \equiv(u, d)$ & $(3,2,1 / 6)$ & $n_{1}$ \\
$u^{c}$ & $\left(3^{*}, 1,-2 / 3\right)$ & $n_{2}$ \\
$d^{c}$ & $\left(3^{*}, 1,1 / 3\right)$ & $n_{3}$ \\
$L \equiv(\nu, e)$ & $(1,2,-1 / 2)$ & $n_{4}$ \\
$e^{c}$ & $(1,1,1)$ & $n_{1}+n_{3}-n_{4}$ \\
\hline$\Phi_{1} \equiv\left(\phi_{1}^{0}, \phi_{1}^{-}\right)$ & $(1,2,-1 / 2)$ & $-n_{1}-n_{3}$ \\
$\Phi_{2} \equiv\left(\phi_{2}^{+}, \phi_{2}^{0}\right)$ & $(1,2,1 / 2)$ & $-n_{1}-n_{2}$ \\
\hline
\end{tabular}

Without $U(1)_{X}$, the following terms are allowed:

$$
L \Phi_{2}, \quad L L e^{c}, \quad L Q d^{c}, \quad u^{c} d^{c} d^{c}
$$

thereby violating both lepton number $(L)$ and baryon number $(B)$. In the MSSM, they are eliminated by the imposition of $R$ parity, i.e. $R \equiv(-)^{3 B+L+2 j}$. Suppose $U(1)_{X}$ is used instead, then

$$
n_{1}=n_{4}=0, \quad n_{2}=-n_{3}
$$

forbids these terms, and satisfies all the anomaly-free conditions for $U(1)_{X}$ to be a gauge symmetry except one, i.e. $\left[U(1)_{X}\right]^{3}$, which reads

$$
3\left[6 n_{1}^{3}+3 n_{2}^{3}+3 n_{3}^{3}+2 n_{4}^{3}+\left(n_{1}+n_{3}-n_{4}\right)^{3}\right]+2\left(-n_{1}-n_{3}\right)^{3}+2\left(-n_{1}-n_{2}\right)^{3}=-3 n_{2}^{3} .
$$

To cancel this contribution, the usual solution is to introduce 3 neutral singlet fermions with $n_{2}$ as their $U(1)_{X}$ assignment, i.e. the canonical minimal extension with three right-handed 
singlet neutrinos where $U(1)_{X}$ is identified as $T_{3 R}$ and $n_{2}=-1 / 2$. Here a different solution is proposed so that the dark scalar doublet model of radiative neutrino mass [1, 2, 3] may be realized. Two doublet superfields and several singlet superfields are added, as shown in Table 2 .

Table 2: New particle content of proposed model.

\begin{tabular}{|c|c|c|}
\hline Superfield & $S U(3)_{C} \times S U(2)_{L} \times U(1)_{Y}$ & $U(1)_{X}$ \\
\hline$\eta_{1} \equiv\left(\eta_{1}^{0}, \eta_{1}^{-}\right)$ & $(1,2,-1 / 2)$ & $n_{2} / 4$ \\
$\eta_{2} \equiv\left(\eta_{2}^{+}, \eta_{2}^{0}\right)$ & $(1,2,1 / 2)$ & $-n_{2} / 4$ \\
\hline$N_{i}$ & $(1,1,0)$ & $n_{2} / 4$ \\
$\chi$ & $(1,1,0)$ & $-3 n_{2} / 4$ \\
\hline$S$ & $(1,1,0)$ & $-n_{2} / 2$ \\
$\zeta$ & $(1,1,0)$ & $3 n_{2} / 2$ \\
\hline
\end{tabular}

Consequently, the $\left[U(1)_{X}\right]^{3}$ anomaly is given by

$$
3(-1)^{3}+2\left(\frac{1}{4}\right)^{3}+2\left(-\frac{1}{4}\right)^{3}+n_{N}\left(\frac{1}{4}\right)^{3}+\left(-\frac{3}{4}\right)^{3}+\left(-\frac{1}{2}\right)^{3}+\left(\frac{3}{2}\right)^{3}=\left(n_{N}-11\right)\left(\frac{1}{4}\right)^{3},
$$

together with the mixed gravitational-gauge $U(1)_{X}$ anomaly

$$
3(-1)+2\left(\frac{1}{4}\right)+2\left(-\frac{1}{4}\right)+n_{N}\left(\frac{1}{4}\right)+\left(-\frac{3}{4}\right)+\left(-\frac{1}{2}\right)+\left(\frac{3}{2}\right)=\left(n_{N}-11\right)\left(\frac{1}{4}\right),
$$

where $n_{N}$ is the number of $N$ superfields. It is clear that $n_{N}=11$ is the simplest solution for an anomaly-free $U(1)_{X}$ in this case. From the assignments of Table 2, there are now the following allowed terms:

$$
L \eta_{2} N, \quad \Phi_{1} \eta_{2} \chi, \quad \eta_{1} \eta_{2}, \quad N N S, \quad \chi \chi \zeta
$$

The one-loop radiative generation of neutrino mass is then possible, as shown in Fig. 2. Assuming that $U(1)_{X}$ is broken spontaneously by the nonzero vacuum expectation values of $S$ and $\zeta$, a discrete $Z_{2}$ symmetry remains, under with $N, \chi, \eta$ are odd, and all other 
superfields are even. This allows a linear combination of $\eta_{1,2}^{0}$ and $\chi$ (call it $\chi_{0}$ ) to be a dark-matter candidate in addition to the lightest neutralino. As for the usual $R$ parity, it remains in effect by assigning $N$ to be odd under $(-)^{L}$ and all other new superfields to be even. Note also that because there are only three families of $L$, only three out of the eleven $N$ 's couple to $L$ through $\eta_{2}$.
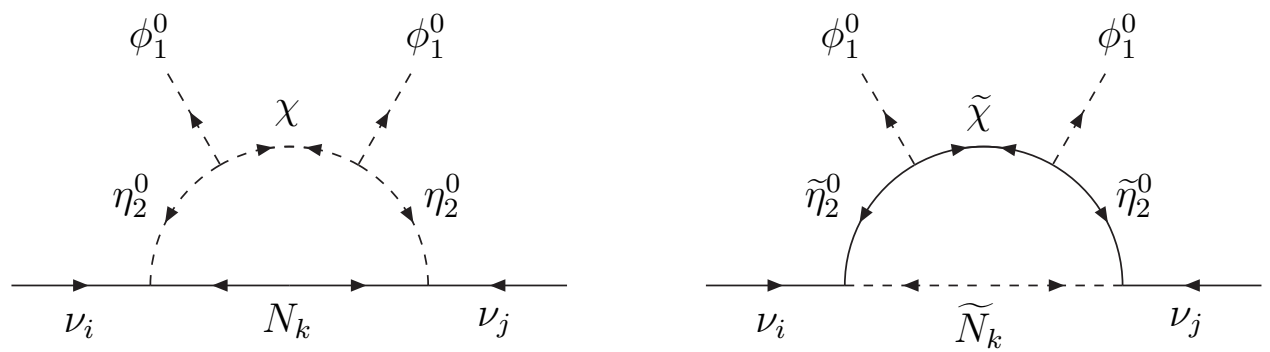

Figure 2: One-loop radiative contributions to neutrino mass.

Because of the two separately conserved discrete symmetries, there are now at least two absolutely stable particles [3, 18, 19, 20, 21]: the lightest particle with $R=-1$ as in the MSSM, and the lightest particle which is odd under the $Z_{2}$ remnant of $U(1)_{X}$. Consider in particular the three lightest particles with $\left(R, Z_{2}\right)=(-,+),(+,-)$, and $(-,-)$ respectively. If one is heavier than the other two combined, then the latter are the two components of dark matter. If not, then all three contribute.

With the particle content of Table 2, the question of fermion masses requires some discussion. It is clear that $\eta_{1} \eta_{2}$ is an allowed mass term, whereas $N$ and $\chi$ obtain Majorana masses through the $N N S$ and $\chi \chi \zeta$ couplings, as $S$ and $\zeta$ acquire nonzero vacuum expectation values. As for $\widetilde{S}$ and $\widetilde{\zeta}$ themselves, a Dirac mass term links the linear combination $\left(3 v_{\zeta} \widetilde{\zeta}-v_{S} \widetilde{S}\right) / \sqrt{9 v_{\zeta}^{2}+v_{S}^{2}}$ to the $U(1)_{X}$ gaugino, whereas $\left(3 v_{\zeta} \widetilde{S}+v_{S} \widetilde{\zeta}\right) / \sqrt{9 v_{\zeta}^{2}+v_{S}^{2}}\left(\right.$ call it $\left.\widetilde{S_{0}}\right)$ remains massless at tree level. To remedy this situation, one way is to add two singlet superfields $\delta_{1,2} \sim \pm 3 n_{2}$, then $\delta_{1} \delta_{2}$ and $\zeta \zeta \delta_{2}$ are allowed terms, and there will be no massless particle at tree level. On the other hand, even without adding anything, $\widetilde{S}$ and $\widetilde{\zeta}$ have 
radiative mass terms in one loop as shown in Fig. 3. The $4 \times 4$ neutralino mass matrix
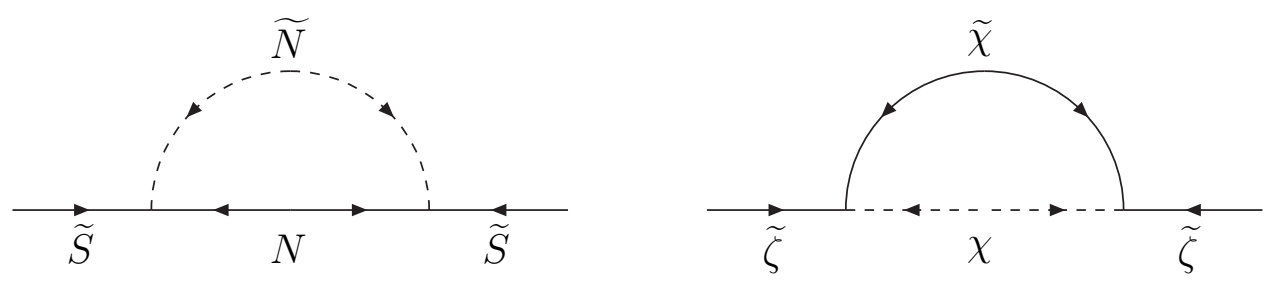

Figure 3: One-loop radiative contributions to $S$ and $\zeta$ Majorana masses.

of the MSSM is then extended to a $7 \times 7$ matrix, with the inclusion of the $U(1)_{X}$ gaugino as well as the $\widetilde{S}$ and $\widetilde{\zeta}$ higgsinos, and $\widetilde{S_{0}}$ is expected to be the lightest neutralino and the dark-matter candidate with $\left(R, Z_{2}\right)=(-,+)$. However, it interacts with quarks through the $U(1)_{X}$ gauge boson with the effective interaction $6\left(v_{\zeta}-v_{S}\right)\left(9 v_{\zeta}^{2}+v_{S}^{2}\right)^{-3 / 2}$ which has to be suppressed by a factor of about $10^{-4}$ relative to $G_{F}$ for it to satisfy the present upper bound on the direct detection of dark matter in underground laboratory experiments. This may be accomplished for example with $v_{\zeta} \sim v_{S} \sim 10 G_{F}^{-1 / 2}$ and $v_{\zeta}-v_{S} \sim G_{F}^{-1 / 2}$. Since there are eleven $N$ 's, the radiative mass of $\widetilde{S}$ in Fig. 3 could be enhanced by an order of magnitude, and for $v_{S} \sim 1 \mathrm{TeV}, m_{\widetilde{S_{0}}}$ may be as high as $100 \mathrm{GeV}$. In that case, $\widetilde{S_{0}} \widetilde{S_{0}}$ may annihilate to $\chi_{0} \chi_{0}$ through the $\chi \chi \zeta$ coupling more efficiently than through its supressed coupling to the $U(1)_{X}$ gauge boson itself.

Since the $U(1)_{X}$ gauge boson $X$ interacts with both quarks and leptons, its production is similar to that of other $\mathrm{U}(1)$ gauge bosons, such as those from $E_{6}$. As already mentioned, as far as the quarks and charged leptons are concerned, $U(1)_{X}$ is identical to the $I_{3 R}$ of $S U(2)_{R}$ with $n_{2}=-1 / 2$. If kinematically alowed, it will be discovered at the LHC. Its possible decay into the new particles of Table 2 will then be a good signature [8] of this model.

To summarize, it has been shown that the dark scalar doublet model of radiative neutrino mass has a supersymmetric realization with the addition of a new $U(1)_{X}$ gauge symmetry. 
Here $R$ parity is an automatic consequence of the MSSM particle content under $U(1)_{X}$. In addition, the spontaneous breaking of $U(1)_{X}$ results in an exactly conserved $Z_{2}$ discrete symmetry which is the basis of the connection between radiative neutrino mass and dark

matter. Taken together, this implies at least two types of dark matter. The new particles of this model, especially the $U(1)_{X}$ gauge boson, are accessible to experimental verification at the LHC.

This work was supported in part by the U. S. Department of Energy under Grant No. DEFG03-94ER40837.

\section{References}

[1] E. Ma, Phys. Rev. D73, 077301 (2006).

[2] E. Ma, Mod. Phys. Lett. A21, 1777 (2006).

[3] E. Ma, Annales de la Fondation de Broglie 31, 285 (2006) hep-ph/0607142].

[4] N. G. Deshpande and E. Ma, Phys. Rev. D18, 2574 (1978).

[5] R. Barbieri, L. J. Hall, and V. S. Rychkov, Phys. Rev. D74, 015007 (2006).

[6] L. Lopez Honorez, E. Nezri, J. F. Oliver, and M. H. G. Tytgat, JCAP 02, 028 (2007).

[7] M. Gustafsson, E. Lundstrom, L. Bergstrom, and J. Edsjo, Phys. Rev. Lett. 99, 041301 (2007).

[8] Q.-H. Cao, E. Ma, and G. Rajasekaran, Phys. Rev. D76, 095011 (2007).

[9] L. M. Krauss, S. Nasri, and M. Trodden, Phys. Rev. D67, 085002 (2003).

[10] K. Cheung and O. Seto, Phys. Rev. D69, 113009 (2004). 
[11] J. Kubo, E. Ma, and D. Suematsu, Phys. Lett. B642, 18 (2006).

[12] J. Kubo and D. Suematsu, Phys. Lett. B643, 336 (2006).

[13] T. Hambye, K. Kannike, E. Ma, and M. Raidal, Phys. Rev. D75, 095003 (2007).

[14] N. Sahu and U. Sarkar, Phys. Rev. D76, 045014 (2007).

[15] K. S. Babu and E. Ma, arXiv:0708.3790 [hep-ph].

[16] E. Ma, arXiv:0708.3371 [hep-ph].

[17] E. Ma, arXiv:0710.1102 [hep-ph].

[18] E. Ma, arXiv:0710.2325 [hep-ph].

[19] E. Ma and U. Sarkar, Phys. Lett. B653, 288 (2007).

[20] T. Hur, H. S. Lee, and S. Nasri, arXiv:0710.2653 [hep-ph].

[21] Q.-H. Cao, E. Ma, J. Wudka, and C.-P. Yuan, arXiv:0711.3881 [hep-ph]. 